\title{
Indicial Response of the Electric Resistance Thermometer Composed of Three Concentric Cylinders
}

\author{
by \\ Kiyotaka Shibata \\ Meteorological Research Institute, Tsukuba, Ibaraki, 305 Japan
}

(Received May 11, 1990 ; Revised June 20, 1990)

\begin{abstract}
To account for the experimental characteristic of the Platinum electric resistance thermometer that it does not follow a first order system in usual meteorological conditions, the indicial response of a model for the electric resistance thermometer is obtained by analytically solving its thermal diffusion equation. The model is so simplified as to be composed of three infinite concentric cylinders, i.e., protector sheath, filling material and electric resistance element. The theoretical indicial response is evaluated for various model thermometers with different properties in the filling material. The major results are as follows: (1) over a certain Fourier number the indicial response can be approximated with one term of the exponential function $C \exp (-t / \lambda)$ including two parameters, coefficient $C(>1)$ and exponent $\lambda ;(2)$ when the Biot number is small, the response speed hardly depends upon the inner structure. It becomes, however, sensitive to the thickness and thermal properties of the filling material as the Biot number increases; (3) the thermal properties of protector sheath and electric resistance element have little effect on the response speed, so long as the filling material is thermally insulating.
\end{abstract}

\section{Introduction}

The governing equation of a thermometer is, in the strict sence, the thermal diffusion equation, i.e., first order derivative as to the time variable and second order as to the spatial variables. However, in ordinary meteorological use the inner structure of the thermometer is neglected for simplicity and so are the spatial variables, and thereby the thermometer is approximated to be described by a linear first order differential equation with respect to time (see e.g., Middleton and Spilhaus, 1953 ; Meteorological Office,
1956). Such an approximation to the linear first order system, i.e., Newtonian heating (or cooling), means that the dynamic response of the thermometer depends upon only one quantity, time constant (e-folding time). For some cases, however, the linear first order system is invalid in describing the thermometer's response.

When a thermometer is subjected to large radiative heating, the Newtonian heating does not obey the linear first order differential equation because of the non-linear heating term. However, we do not treat the non-linear first order system like this, but 
we investigate the linear yet non-first order system due to the inner structure itself. The criteria of the approximation to the first order system are calculated by Heisler (1947) for simple structures of the oneconstituent system such as sphere, cylinder or plate, but it has not yet been calculated for complex structures of multi-constituent systems.

The electric resistance thermometer, in which a metal electric resistance wire is ordinarily put in a cylindrical metal protector sheath, is neither a simple structure nor a one-constituent system. Notwithstanding these facts the approximation of the first order system is usually applied to the electric resistance thermometer a priori (see e.g., Middleton and Spilhaus, 1953 ; Meteorological Office, 1956). The experiment carried out by Tokuue et al. (1984), however, indicated that the indicial response of the electric resistance thermometer does not coincide with that of the first order system in usual meteorological conditions.

In this paper we theoretically account for this experimental result by using much simplified model thermometers. We assume that the electric resistance thermometer is composed of three concentric cylinders of infinite length, i.e., protector sheath, filling material and electric resistance element, and obtain its indicial response by analytically solving its thermal diffusion equation. In addition, we set various thermometers in order to investigate the effects of the protector sheath, filling material and electric resistance element on the response speed of the thermometer, and compare them with the experiment by Tokuue et al. (1984).

\section{Dynamic characteristics}

Tokuue et al. (1984) measured the indicial response of the Platinum electric resistance thermometer by decreasing the ambient temperature stepwise, in which experiment two ambient materials, air and water, were used. Fig. 1 shows the experimental result of the response of the thermometer. The disper- sion of data is greater in water than in air, because the thermometer was stirred by hand in water, which treatment caused the relative water speed to vary widely in each trial. On the other hand, air was blown at constant speed parallel to the long axis of the thermometer in the air. Considering the accuracy of measurements, we pick out the thermometer which Tokuue et al. (1984) measured repeatedly. Then the e-folding times are summarised in the following way.

Characteristic-1. The Platinum electric resistance thermometer of protector sheath $10 \mathrm{~mm} \times 150 \mathrm{~mm}$ (diameter $\times$ length) has the e-folding times of 140 sec in air (wind speed $6 \mathrm{~m} / \mathrm{s}$ ) and $33 \mathrm{sec}$ in water (water speed unknown), respectively.

If the approximation to a first order system is valid, the dimensionless temperature ratio $\Theta$ can be represented by the equation, $\ln \Theta=-t / \tau$, where $\tau$ is the e-folding time. Nevertheless, the equation does not agree well with the experiment as seen in Fig. 1. We then calculate the best-fit lines for the experimental data using the weight $\Theta$ to lessen the dispersion caused by the logarithmic coordinate. The result is summarised as:

Charactrristic-2. When $t / \tau>1 / 5$, the best-fit lines in air and in water, respectively, are

$\ln \Theta=-1.11 t / \tau+\ln 1.12, \sigma=0.043$, in air, $\ln \Theta=-1.10 t / \tau+\ln 1.11, \sigma=0.040$, in water, where $\sigma$ represents the standard error.

In Characteristic- 2 the e-folding time of Fig. 1 is multiplied by 0.97 so that $\ln \Theta=-1$ at $t=\tau$, and the same correction is made in Characteristic- 1 . That is, we redetermine $\tau$ from the best-fit line. When $t / \tau<1 / 5$, the data are too scarce and coase to get the bestfit lines, but we can derive a qualitative feature from Fig. 1 ; the response is by far slower than that of a first order system. (There scarcely seems temperature decrease in the raw record. (see Tokuue et al., (1984))

\section{Mathematical analysis}

The actual Platinum electric resistance thermometer has a very complicated inner 


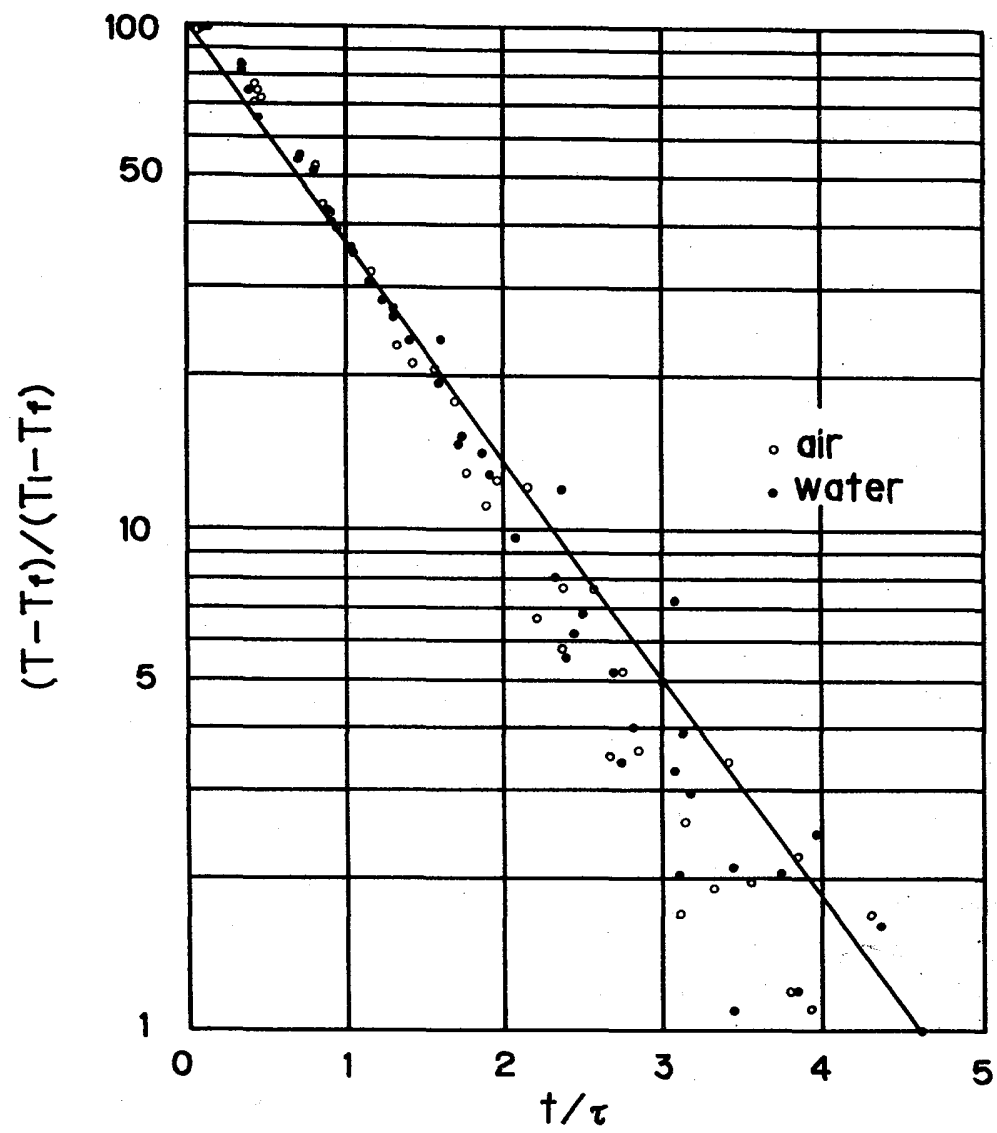

Fig. 1. Indicial response of the Platinum electric resistance thermometer. Open and solid circles are the experimental values in air and water, respectively. The solid line represents $\ln \Theta=-t / \tau$. (after Tokuue et al., 1984)

structure ; for instance, the electric resistance element, which consists of a coil sandwiched with a number of electrically insulating sheets like mica, is framed by a metal and plugged into a stainless steel cylindrical protector sheath. We, however, consider intuitively that the response characteristics are determined, to a large extent, by filling material and, to a lesser extent, by the procector sheath and inner materials including a resistance element. Therefore, in mathematical models we treat the inner materials as one material, i.e., electric resistance element and assume that the electric resistance thermometer consists of three concentric cylinders of electric resistance element, filling material, and protector sheath.
Let suffixes 1, 2, 3 refer to electric resistance element, filling material, protector sheath, respectively, and let the thermal diffusivity be $\kappa$, thermal conductivity $K$, outer radius of each material $a, b, c\left(=r_{1}, r_{2}, r_{3}\right)$, the initial and ambient temperatures $T_{i}$ and $T_{f}$, respectively. The boundary condition at $r=c$ is the radiation type, where the mean thermal emissivity is represented by $h$. First we investigate the end effect of a finite cylinder. Consider the indicial response of a solid cylinder of radius $c$ and length $2 l$. The boundary conditions at both ends are of two types: (1) one end is radiative and the other insulating; and (2) both ends are radiative. The former type (1), however, becomes equivalent to the latter type when the length 
is doubled, and hence we treat here only the

latter type. The solution is given by

$$
\Theta(z, r, t)=R(r, t) \sum_{m} \frac{2\left(K^{2} g_{m}{ }^{2}+h^{2} l^{2}\right) \sin g_{m}}{g_{m}\left(K^{2} g_{m}{ }^{2}+K h l+h^{2} l^{2}\right)} \cos \left(\frac{g_{m} z}{l}\right) \exp \left(-\frac{\kappa g_{m}{ }^{2}}{l^{2}} t\right)
$$

with the eigenvalue equation

$$
\frac{K}{h l}=\frac{\cot g_{m}}{g_{m}},
$$

where $R(r, t)$ is the solution of a cylinder of infinite length (see e.g., Carslaw and Jaeger, 1959). The end effect appears in coefficients and exponents. Since we evaluate the end effect not locally but globally, we average the coefficients with respect to the whole length. When the length is much larger than the radius, $l \gg c$, the exponent term can be neglected compared with that of the infinite cylinder. On the other hand, the coefficient is almost 1 when the Biot number $(B i)$ of axial direction is very small, $K / h l \ll 1$, while it approaches $8 / \pi^{2}(=0.81)$ when $B i$ increases to infinity. For a solid cylinder of $l \gg c$ therefore the end effect can be represented by

$$
\Theta(r, t)= \begin{cases}R(r, t) & K / h l \ll 1 \\ 0.81 R(r, t) & K / h l \gg 1\end{cases}
$$

It is not easy to analytically evaluate the model thermometer, i.e., a composite finite cylinder, and thereby we assume that the end effect of the model is as negligible as that of

a solid cylinder when $l \gg c$ and $B i$ of axial direction is small. The latter condition is replaced with the condition that $B i$ of radial direction is small, because $B i$ of radial direction is much larger than that of axial direction, $K / h c \gg K / h l$, for $l \gg c$. Since the electric resistance thermometer Tokuue et al. (1984) used can be regarded as $l \gg c$ (see Characteristic-1), and $B i$ 's of radial direction in their experiments can be inferred to be small as will be shown later, we will neglect the end effect of the model. The spatial variable hence becomes only $r$, leading to the governing equations:

$$
\begin{aligned}
& \frac{\partial T_{j}}{\partial t}=\kappa_{j}\left(\frac{\partial^{2}}{\partial r^{2}}+\frac{1}{r} \frac{\partial}{\partial r}\right) T_{j}, \quad j=1,2,3, \\
& T_{j}=T_{i}, \quad \text { when } t=0, \quad j=1,2,3, \\
& T_{j}=T_{j+1}, \quad \text { at } r=r_{j}, \quad j=1,2, \\
& K_{j} \frac{\partial T_{j}}{\partial r}=K_{j+1} \frac{\partial T_{j+1}}{\partial r}, \quad \text { at } r=r_{j}, \quad j=1,2, \\
& K_{3} \frac{\partial T_{3}}{\partial r}=h\left(T_{f}-T_{3}\right), \quad \text { at } r=r_{3}
\end{aligned}
$$

The solutions are (see Appendix)

$$
\begin{aligned}
& \Theta_{1}=-2 \sum_{\mathrm{n}=1}^{\infty} \frac{\omega P\left(a, a, \alpha_{n}\right) P\left(a, a, \mu \alpha_{n}\right) J_{0}\left(\nu \alpha_{n} r\right)}{D N\left(\alpha_{n}\right)} \frac{\exp \left(-\kappa_{2} \alpha_{n}{ }^{2} t\right)}{\alpha_{n}} \\
& \Theta_{2}=-2 \sum_{\mathrm{n}=1}^{\infty} \frac{P\left(b, b, \nu \alpha_{n}\right) V\left(r, a, \alpha_{n} r\right)}{D N\left(\alpha_{n}\right)} \frac{\exp \left(-\kappa_{2} \alpha_{n}{ }^{2} t\right)}{\alpha_{n}} \\
& \Theta_{3}=-2 \sum_{n=1}^{\infty} \frac{U\left(r, \alpha_{n}\right)}{D N\left(\alpha_{n}\right)} \frac{\exp \left(-\kappa_{2} \alpha_{n}{ }^{2} t\right)}{\alpha_{n}}
\end{aligned}
$$

with the eigenvalue equation

$$
\frac{K_{3}}{h c} \mu c \alpha_{n}=\frac{V\left(\alpha_{n}\right) P\left(c, b, \mu \alpha_{n}\right)-\sigma W\left(\alpha_{n}\right) O\left(c, b, \mu \alpha_{n}\right)}{V\left(\alpha_{n}\right) P\left(c, b, \mu \alpha_{n}\right)+\sigma W\left(\alpha_{n}\right) O\left(c, b, \mu \alpha_{n}\right)},
$$

where 


$$
\begin{aligned}
& \mu=\sqrt{\kappa_{2} / \kappa_{3}}, \quad \sigma=K_{2} / K_{3} / \mu, \quad \nu=\sqrt{\kappa_{2} / \kappa_{1}}, \quad \omega=K_{2} / K_{1} / \nu, \\
& O(x, y, z)=J_{0}(x z) Y_{0}(y z)-Y_{0}(x z) J_{0}(y z), \\
& P(x, y, z)=J_{0}(x z) Y_{1}(y z)-Y_{0}(x z) J_{1}(y z), \\
& Q(x, y, z)=J_{1}(x z) Y_{1}(y z)-Y_{1}(x z) J_{1}(y z), \\
& S(z)=(1-\nu \omega) J_{1}(\nu z a) P(b, a, z)+(\omega-\nu) J_{0}(\nu z a) O(b, a, z), \\
& T(z)=(1-\nu \omega) J_{1}(\nu z a) Q(b, a, z)-(\omega-\nu) J_{0}(\nu z a) P(a, b, z), \\
& V(x, y, z)=\omega J_{0}(\nu y z) P(x, y, z)-J_{1}(\nu y z) O(x, y, z), \quad V(z)=V(b, a, z), \\
& W(x, y, z)=\omega J_{0}(\nu y z) Q(x, y, z)-J_{1}(\nu y z) P(x, y, z), \quad W(z)=W(b, a, z), \\
& U(r, z)=V(z) P(r, b, \mu z)-\sigma W(z) O(r, b, \mu z), \\
& D N(z)=[\{a S(z)-b W(z)\} P(c, b, \mu z)-\sigma\{a T(z)+b V(z)\} O(c, b, \mu z) \\
& \quad-\mu\{c Q(c, b, \mu z)-b O(b, c, \mu z)\} V(z)+\sigma \mu\{b P(c, b, \mu z)-c P(b, c, \mu z)\} W(z)] \\
& \quad-\frac{K_{3}}{h c} \mu c z[\{a S(z)-b W(z)\} Q(c, b, \mu z)+\sigma\{a T(z)+b V(z)\} P(b, c, \mu z) \\
& \quad-\mu\{c P(c, b, \mu z)-b P(b, c, \mu z)\} V(z)+\sigma \mu\{b Q(c, b, \mu z)-c O(c, b, \mu z)\} W(z)],
\end{aligned}
$$

$J_{0}$ and $J_{1}$ are the Bessel functions of the first kind of order 0 and 1 , respectively, and $Y_{0}$ and $Y_{1}$ those of the second kind of order 0 and 1 , respectively.

All the solutions of Eqs.(6)-(8) can be formally represented by

$$
\Theta=\sum_{n=1}^{\infty} C_{n} \exp \left(-\frac{t}{\lambda_{n}}\right)
$$

where $C_{n}$ is a function of $r$ and $\lambda_{n}=\left(\kappa_{2} \alpha_{n}{ }^{2}\right)^{-1}$. $\Theta$ is then approximated by a finite sum of exponential functions according to the time intervals as

$$
\begin{array}{r}
\Theta=C_{1} \exp \left(-\frac{t}{\lambda_{1}}\right), \quad t_{1}<t<\infty, \\
\Theta=C_{1} \exp \left(-\frac{t}{\lambda_{1}}\right)+C_{2} \exp \left(-\frac{t}{\lambda_{2}}\right), \\
t_{2}<t<t_{1},
\end{array}
$$

$$
\begin{array}{r}
\Theta=C_{1} \exp \left(-\frac{t}{\lambda_{1}}\right)+\cdots+C_{n} \exp \left(-\frac{t}{\lambda_{n}}\right), \\
t_{n}<t<t_{n-1},
\end{array}
$$

where $t_{n}$ is the time beyond which the solution is represented by a sum of $n$ eigenfunctions with reasonable precision. If the efolding time $\tau$ is larger than $t_{1}$ as in Fig. 1, then $\tau$ takes a simple form:

$$
\tau=\left(1+\ln C_{1}\right) \lambda_{1} \text {. }
$$

\section{Numerical calculation of the models' re- sponses}

\subsection{Parameter setting}

Making the radius of each cylinder nondimensional form with respect to the outer radius of the protector sheath, " $c$ ", the eigenvalue becomes $c \alpha_{n}$, and Eqs.(6)-(8) come to include 6 parameters, $a / c, b / c, \mu, \sigma, \nu$, and 
$\omega$. Since the details of the electric resistance thermomener Tokuue et al.(1984) used is not described, we set the following thermometer as a prototype thermometer (I): The outer radius and thickness of the protector sheath are $5 \mathrm{~mm}$ and $1 \mathrm{~mm}$, respectively, and the thickness of the filling material is $0.5 \mathrm{~mm}$, leading to $a / c=0.7$ and $b / c=0.8$. To decrease the number of parameters, we set the electric resistance element to be composed of the same material as the protector sheath, i.e., $\omega=\sigma, \nu=\mu$. This setting is, as will be shown later, based upon the fact that the response speed hardly depends upon the thermal properties of the protector sheath and resistance element, but mainly upon that of the filling material, when the filling material is not so good a thermal conductor. The protector sheath is made of 18.8 stainless steel (Ni: $18 \%, \mathrm{Cr}: 8 \%$ ), and the filling material is composed of silicon grease for heat sink (air is also used as the filling material in another thermometer). Table 1 shows the physical properties of air, and silicon grease, and $18 \cdot 8$ stainless steel, respectively.

To investigate the effects of the properties of each cylinder on the response characteristics of the model thermometer, we also set various thermometers (II)-(XIII). The thermometers (II)-(VII) in Table 2 have silicon grease of various thermal properties but the same thickness in filling materials. The set of $(\sigma, \mu)$ or $(\tau, \nu)$ in the thermometers (II)-(VII) is multiplied or divided by $\sqrt{2}$ of that in the thermometer (I). The effect of the thickness of the filling material is studied, by changing the parameter $a / c$, in the thermometers (VIII)-(XIII) as shown in Table 3, where the thermometers (VIII) and (IX) have silicon grease fillings and the ones (X)-(XIII) have air fillings.

\subsection{Characteristics of the prototype thermometer (I)}

Fig. 2 shows the graph of $B i\left(=K_{3} / h c\right)$ vs $\left(\mu c \alpha_{1}\right)^{-2}$ for some model thermometers and a solid cylinder, in which $\omega=\sigma=\nu=$ $\mu=1$. As $\lambda_{1}=c_{2} \kappa_{3}^{-1}\left(\mu c \alpha_{1}\right)^{-2}, \lambda_{1}$ can be obtained by multiplying the scale of coordinate in Fig. 2 by $c^{2} \kappa_{3}^{-1}$. (Multiplication of
5.6 yields $\lambda_{1}$ in unit of second for the $18 \cdot 8$ stainless steel.) Comparisons between the experiment and the model will be made in terms of $C_{1}$ and $\lambda_{1}$, because these two quantities can describe the experimental results with sufficient accuracy (see Characteristic2).

We can derive important features of $\lambda_{1}$ by examining the dependence of $\lambda_{1}$ on $B i$ in Fig. 2. The entire $B i$ region can be appoximately separated into three regions: When $B i<0.1,\left(\mu c \alpha_{1}\right)^{-2} \propto B i$, so that $\lambda_{1}^{-1} \propto c / h$; similarly, $\lambda_{1}^{-1} \propto\left(h K_{3}\right)^{-1 / 2} c^{3 / 2}$ when $0.1<B i$ $<1.0$; and $\lambda_{1}^{-1} \propto c^{2} K_{3}^{-1}$ when $1.0<B i$. Hence, $\lambda_{1}^{-1}$ does not depend upon the thermal conductivity $K_{3}$ of the outer cylinder in the region $B i<0.1$, and does not upon the thermal emissivity $h$ in the region $B i>1.0$, while $\lambda_{1}^{-1}$ depends upon both quantities, $K_{3}$ and $h$, in the intermediate region of $0.1<B i$ $<1.0$. These features are qualitatively common in the models and a solid cylinder, because the graphs of $B i$ vs $\left(\mu c \alpha_{1}\right)^{-2}$ are conformable to one another as seen in Fig. 2. Differences among them are the values of the three regions of $B i$.

Next we consider the coefficient $C_{1}(r)$, particularly the one in the first cylinder. Our concern consists in the areally averaged value in the resistance element from $r=0$ to

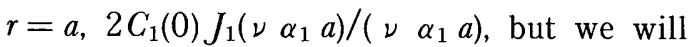
use the center value $C_{1}(0)$. This is because $2 J_{1}\left(\nu \alpha_{1} a\right) /\left(\nu \alpha_{1} a\right)$ is nearly equal to 1 when $B i$ is not so large. Fig. 3 shows the curves of $C_{1}(0)$ for the various model thermometers and a solid cylinder. There is a distinct feature in $C_{1}\left(=C_{1}(0)\right)$ of the model thermometer ; it shows a maximum value for the model thermometers, whereas it monotonically increases with $B i$ for a solid cylinder. Thus the behavior beyond the maximum value exhibits the particular characteristics of the composite cylinder which contains a thermal insulating cylinder. We will refer to the $B i$ at which $C_{1}$ takes the maximum value as the critical Biot number (Bicr).

Fig. 4 shows dimensionless temperature ratios at $r=0$ for the thermometer(I) and a solid cylinder. The time of the one- and two- 
Table 1 Physical properties of air, silicon grease, and $18 \cdot 8$ stainless steel. Density, thermal conductivity, and diffusivity of silicon grease are the arithmetic means of those of three silicon greases for heat sink, KS609, KS612, and G746, made by the Sinetu Chemistry Co. Ltd., and specific heat is calculated from those of their base oils. $\sigma$ and $\mu$ are the values when the third cylinder is made of $18 \cdot 8$ stainless steel.

\begin{tabular}{ccccccc}
\hline & \multicolumn{6}{c}{ Physical Properties } \\
\cline { 2 - 7 } Material & Density & $\begin{array}{c}\text { Specific } \\
\text { Heat }\end{array}$ & $\begin{array}{c}\text { Thermal } \\
\text { Conductivity }\end{array}$ & Diffusivity & & \\
& $\begin{array}{c}\rho \\
\left(\mathrm{kg} / \mathrm{m}^{3}\right)\end{array}$ & $\begin{array}{c}S \\
(\mathrm{kcal} / \mathrm{kgK})\end{array}$ & $\begin{array}{c}K \\
(\mathrm{kcal} / \mathrm{mhrK})\end{array}$ & $\begin{array}{c}\kappa \\
\left(\mathrm{m}^{2} / \mathrm{hr}\right)\end{array}$ & $\sigma$ & $\mu$ \\
\hline Air & 1.17 & 0.24 & 0.022 & 0.079 & $0.71 \times 10^{-4}$ & 0.22 \\
$\begin{array}{c}\text { Silicon } \\
\text { Grease }\end{array}$ & 2500 & 0.36 & 0.54 & $0.6 \times 10^{-5}$ & 0.2 & 0.19 \\
$\begin{array}{c}\text { Stainless } \\
\text { Steel }\end{array}$ & 7800 & 0.11 & 14.0 & 0.016 & & \\
\hline
\end{tabular}

Table 2 Parameters for thermometers (I) (VII). The second cylinders in all these thermometers are of silicon grease. The last two rows show the ratios of thermal conductivity to that of (I) when $K_{2}$ or $K_{3}$ is fixed.

\begin{tabular}{|c|c|c|c|c|c|c|c|}
\hline \multirow{2}{*}{$\begin{array}{c}\text { Param- } \\
\text { eter }\end{array}$} & \multicolumn{7}{|c|}{ Type } \\
\hline & I & II & III & $\mathrm{N}$ & $\mathrm{V}$ & $\mathrm{VI}$ & VII \\
\hline$a / c$ & .70 & .70 & .70 & .70 & .70 & .70 & .70 \\
\hline$b / c$ & .80 & .80 & .80 & .80 & .80 & .80 & .80 \\
\hline$\sigma$ & .20 & .28 & .20 & .28 & .14 & .20 & .14 \\
\hline$\mu$ & .19 & .27 & .19 & .27 & .13 & .19 & .13 \\
\hline$\tau$ & .20 & .20 & .28 & .28 & .20 & .14 & .14 \\
\hline$\nu$ & .19 & .19 & .27 & .27 & .19 & .13 & .13 \\
\hline \multicolumn{2}{|c|}{$K_{1} K_{3}\left(K_{2}:\right.$ fix $)$} & $11 / 2$ & $1 / 21$ & $1 / 21 / 2$ & 1 & 2 & 22 \\
\hline \multicolumn{2}{|c|}{$K_{1} K_{2}\left(K_{3}:\right.$ fix $)$} & 22 & $1 / 21$ & $1 \quad 2$ & $1 / 2 \quad 1 / 2$ & 21 & $11 / 2$ \\
\hline
\end{tabular}

Table 3 Parameters for thermometers (I), (VIII) (XIII). The second cylinder is silicon grease in (I), (VIII), and (IX), while it is air in (X) (XIII). The thermal properties of the first and the third are equivalent in all these thermometers.

\begin{tabular}{|c|c|c|c|c|c|c|c|}
\hline \multirow{2}{*}{$\begin{array}{l}\text { Param- } \\
\text { eter }\end{array}$} & \multicolumn{7}{|c|}{ Type } \\
\hline & $I$ & VIII & IX & $X$ & $X I$ & XII & XIII \\
\hline$a / c$ & .70 & .75 & .60 & .78 & .75 & .70 & .60 \\
\hline$b / c$ & .80 & .80 & .80 & .80 & .80 & .80 & .80 \\
\hline$\sigma$ & .20 & .20 & .20 & $.7 \mathrm{E}-3$ & $.7 E-3$ & $.7 \mathrm{E}-3$ & $.7 \mathrm{E}-3$ \\
\hline$\mu$ & .19 & .19 & .19 & $.2 \mathrm{E}+1$ & $.2 \mathrm{E}+1$ & $.2 \mathrm{E}+1$ & $.2 \mathrm{E}+1$ \\
\hline
\end{tabular}




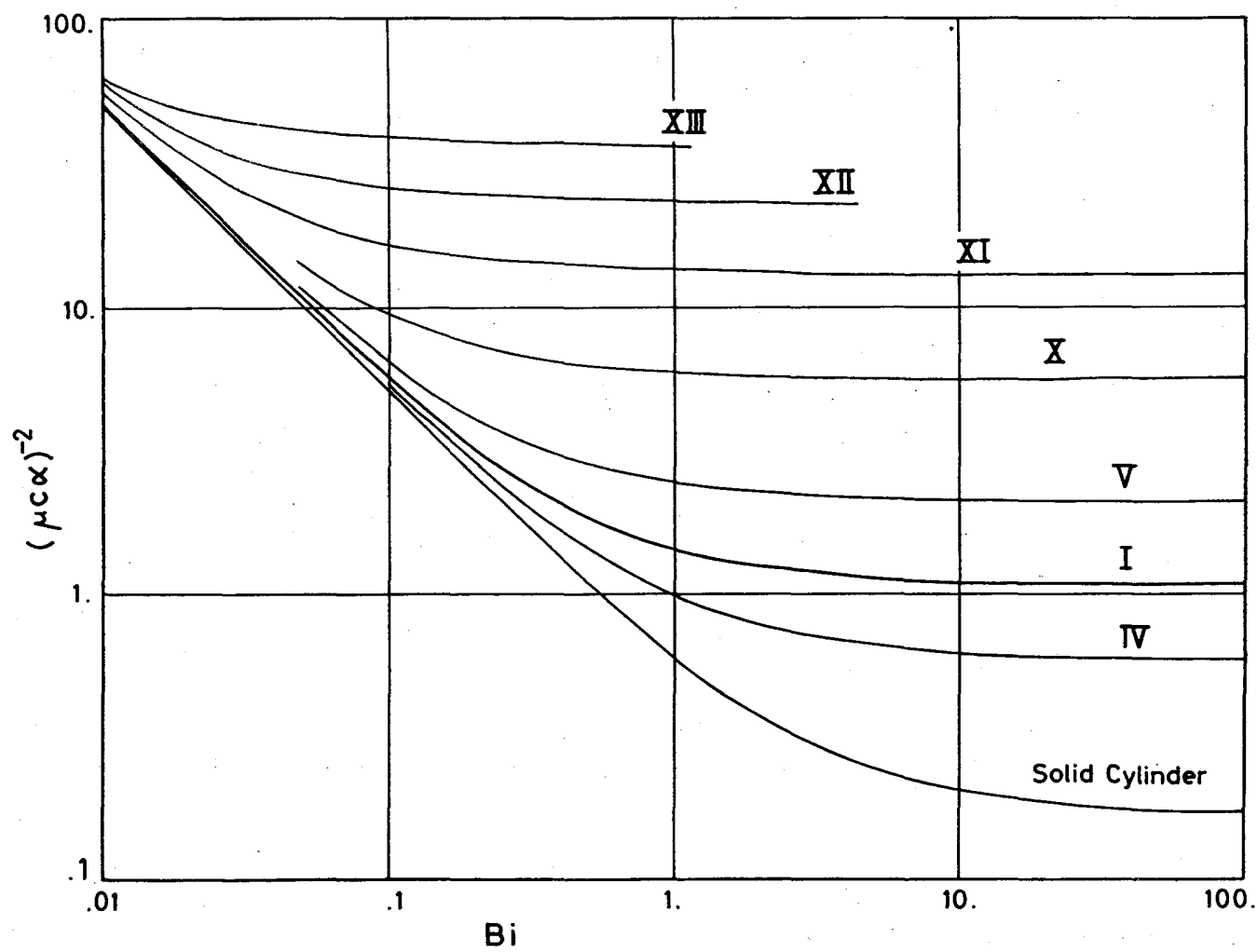

Fig. $2\left(\mu c \alpha_{1}\right)^{2}$ versus $B i$ for various model thermometers.

term approximations, $t_{1}$ and $t_{2}$, the errors of which are within $0.5 \%$, are shown by arrows and triangles, respectively, in Fig. 4 (Multiplication by the e-folding time $\tau$ yields the Fourier number $\left(\kappa_{3}(\mu c)^{2} t\right)$. As the coefficient $C_{1}$ is the intercept of the line of one-term approximation $\ln \Theta(\mathrm{t})=-\left(t-t_{1}\right) / \lambda_{1}+\ln \Theta\left(t_{1}\right)$, $\ln C_{1}=t_{1} / \lambda_{1}+\ln \Theta\left(t_{1}\right)$. Hence, $C_{1}$ becomes large when the inclination $\lambda_{1}^{-1}, t_{1}$ or $\Theta\left(t_{1}\right)$ is large.

In a solid cylinder $t_{1}$ increases very slowly $\left(t_{1}=0.03,0.05,0.05\right.$ for $B i=0.1,1.0$, 10. in Fig. 4), while $\Theta\left(t_{1}\right)$ decreases rapidly $\left(\Theta\left(t_{1}\right)=0.99,0.80,0.45\right.$ for $B i=0.1,1.0$, 10.). However, the rapid increase of $\lambda_{1}^{-1}$ (see Fig. 2) outweighs the decrease of $\Theta\left(t_{1}\right)$ when $0.1<B i<10$, so that $G_{1}$ becomes an increasing function of $B i$ and shows a sharp rise in the region of $0.1<B i<10$. When a thermally insulating cylinder is sandwiched between thermal conducting ones as in the thermom- eter (I), $t_{1}$ and $\Theta\left(t_{1}\right)$ behave differently, though $\lambda_{1}^{-1}$ shows a similar increase (see Fig. 2$) ; t_{1}$ rapidly decreases with $B i\left(t_{1}=\right.$ $0.19,0.12,0.04$ for $B i=0.1,1.0,10$.), while $\Theta\left(t_{1}\right)$ increases after decreasing $\left(\Theta\left(t_{1}\right)=\right.$ $0.89,0.72,0.92$ for $B i=0.1,1.0,10$.). Thus in the small $B i$ region the effect of $\lambda_{1}^{-1}$ is greater than those of $t_{1}$ and $\Theta$, but the effect of $t_{1}$ comes to surpass the others as $B i$ increases, and thereby $C_{1}$ begins to decrease at a certain $B i$. This characteristic of $C_{1}$ comes to approach that of a solid cylinder as the degree of the thermal.insulation of the second cylinder weakens. That is, the critical Biot number (Bicr) increases, and the decrease in $C_{1}$ after the maximum value becomes small.

\subsection{Effects of the thermal conductivity.}

If we assume that the thermometers (I)-(VII) are different only in thermal conductivity, then three cases can be thought 


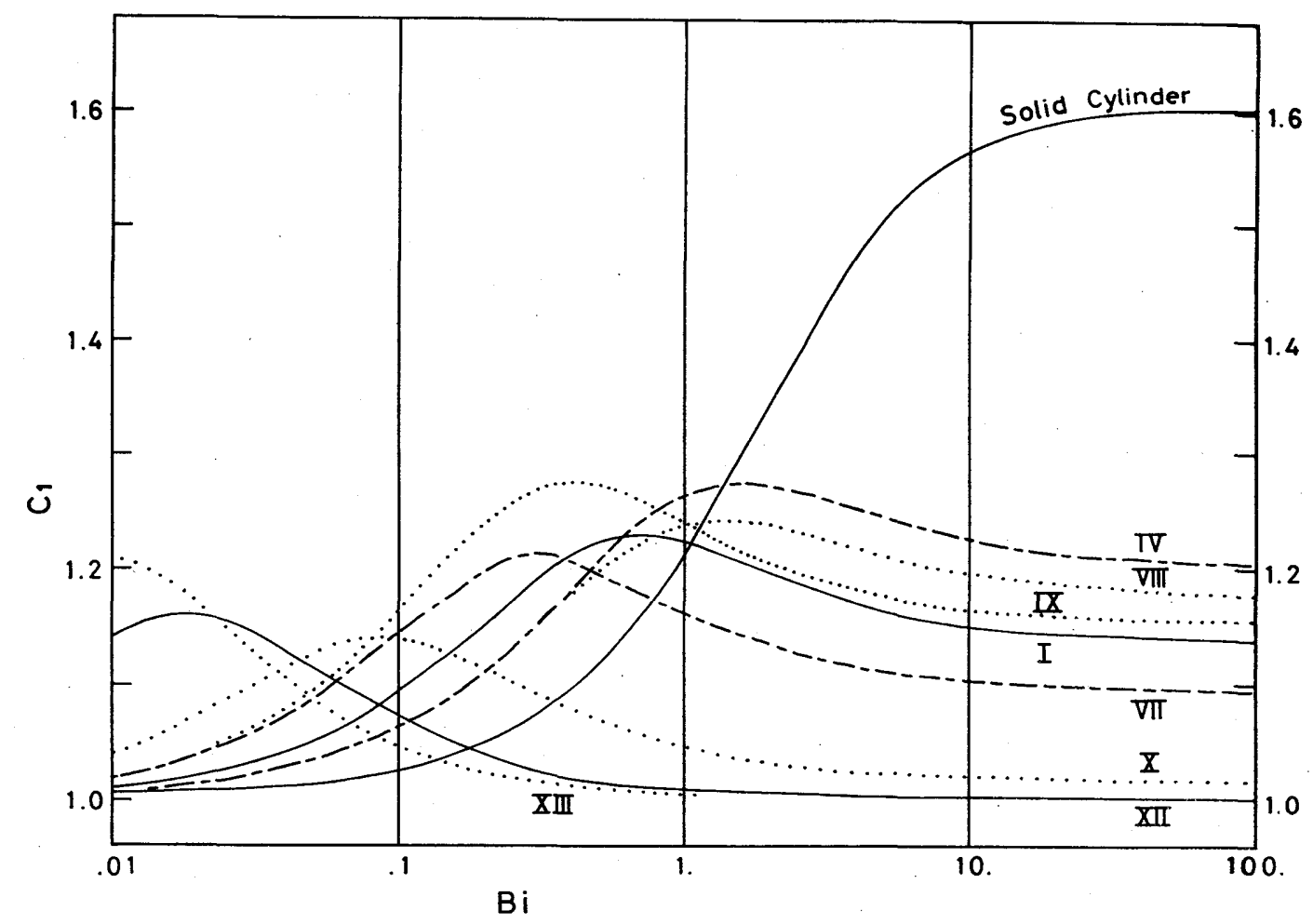

Fig. $3 C_{1}(0)$ versus $B i$ for various model thermometers.

according to which is the fixed thermal conductivity. We treat here only two cases: one is fixed $K_{3}$ and the other is fixed $K_{2}$. We concentrate on $\lambda_{1}$ though the difference of $C_{1}$ among the thermometers (I)-(VII) is not small. This is because the behavior of $C_{1}$ is too complicated to treat as seen in Fig. 3.

(a) When $K_{3}$ is fixed.

In this case the thermal conductivities of the first and second cylinders, $K_{1}$ and $K_{2}$, become twice or half that of the thermometer(I) in every thermometer. The relation of thermometer types to the thermal conductivity ratios are shown in the last row in Table 2 . Since $B i$ is common to all the thermometers owing to fixed $K_{3}$, the order of magnitudes of $\lambda_{1}$ can be obtained easily, (II) $<$ (IV) $<$ (VI) $<$ (I) $<$ (III) $<$ (VII) $<$ (V), where the ratios of $\lambda_{1}$ to that of the thermometer (I) fall within the range $0.998-1.02$ at $B i=0.02 ; 0.942-1.13$ at $B i=0.1$; and $0.677-1.68$ at $B i=1.0$. $\lambda_{1}$ of the thermometer (VI) is greater than that of the thermometer (IV), where the ratios of $K_{1}$ and $K_{2}$ between them are $1 / 2$ and 2 , respectively. The same relation holds between the thermometers (VII) and (III). This indicates that the effect of $K_{2}$ on $\lambda_{1}$ is greater than that of $K_{1}$. In a large $B i$ region $\lambda_{1}$ is determined mainly according to the magnitude of $K_{2}$ as (II) $\sim(\mathrm{IV}) \ll(\mathrm{VI}) \sim(\mathrm{I}) \sim(\mathrm{III})$ $\ll(\mathrm{VII}) \sim(\mathrm{V})$. On the other hand, when $B i<$ 0.05 , there is substantially no difference among the thermometers (I)-(VII).

(b) When $K_{2}$ is fixed.

The thermal conductivities of the first and third cylinders, $K_{1}$ and $K_{3}$, become twice or half that of the thermometer(I) as shown in the second to the last row in Table 2. The order of $\lambda_{1}$ becomes (VII) $<$ (V) $<$ (VI) $<$ (I) $<$ (III) $<$ (II) $<$ (IV). Note that $B i$ is not common for this case. When we take $B i=0.02,0.1$, 1.0 for the thermometer(I), the ratios of $\lambda_{1}$ to 


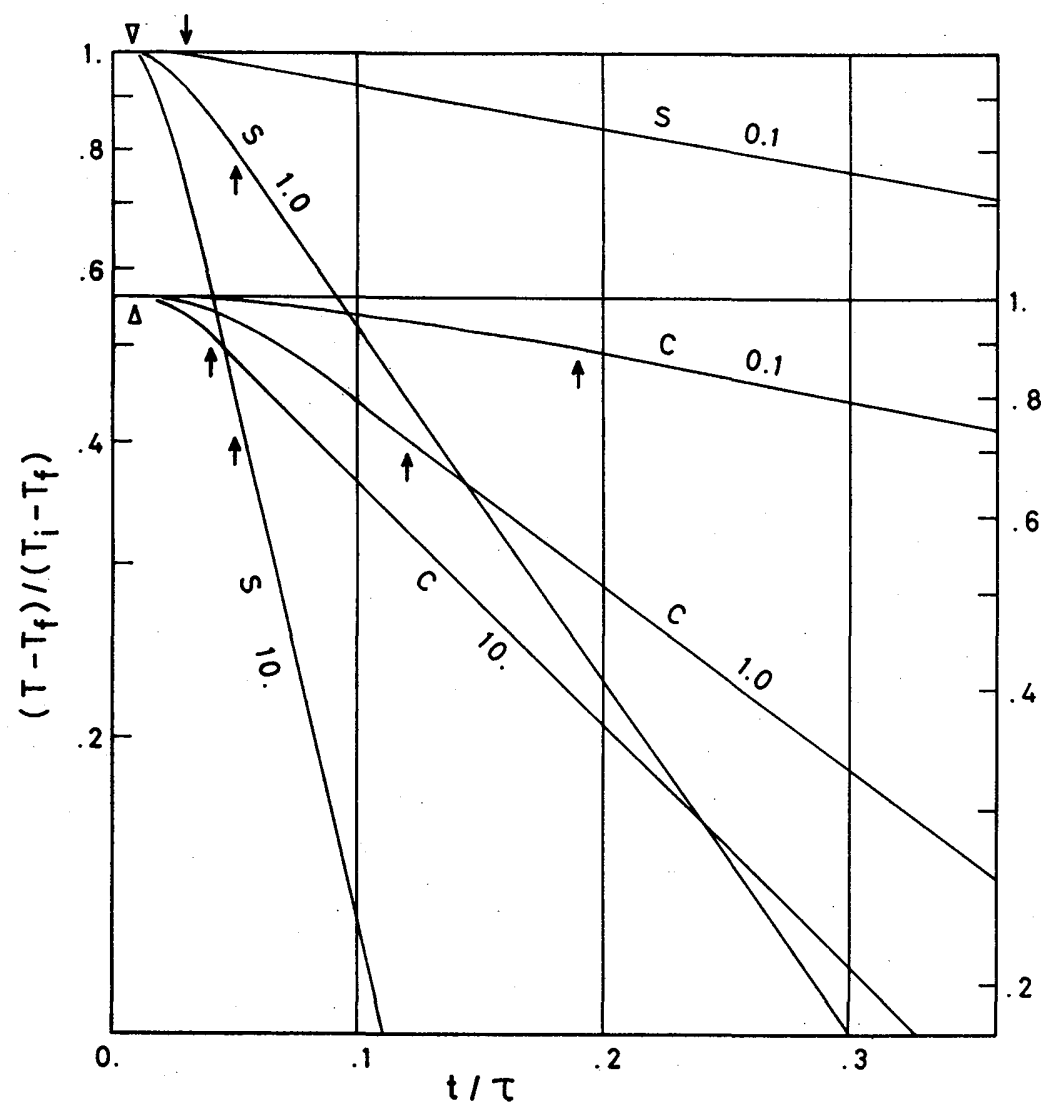

Fig. 4 Dimensionless temperature ratio at $r=0$ for the thermometer (I) ( $C$, right ordinate) and a solid cylinder ( $S$, left ordinate). Numbers near the curves represent $B i$. $\uparrow$ (or $\downarrow$ ) and $\Delta$ (or $\nabla$ ) are the times of one-term and two-term approximations ( $0.5 \%$ error), $t_{1}$ and $t_{2}$ respectively. Time in the absicca is devided by $\tau$ at $B i=0.1$.

that of the thermometer (I) fall within the ranges, $0.998-1.00,0.990-1.02$, and $0.995-1.09$, respectively. The range of the ratios of $\lambda_{i}$ is very small, nearly 1 , for any $B i$, in contrast with when $K_{3}$ is fixed. Also in this case $\lambda_{1}$ is more sensitive to $K_{3}$ that to $K_{1}$, but the difference between the effects of $K_{3}$ and $K_{1}$ is much smaller than that between the effects of $K_{2}$ and $K_{1}$ under fixed $K_{3}$.

\subsection{Effects of the filling material}

We consider here the effect of filling material in comparison with that of thermal conductivity. Consider the thermometers (VII) and (IV). Compared with the thermometer (I), the former is half in thickness in the filling material(silicon grease), and the latter is twice in value in $K_{2}$ (see Table 2 and 3 ). Though $\lambda_{1}$ of the thermometer (VII) is larger than that of the thermometer (VI), the difference in $\lambda_{1}$ is very small; it is less than $1 \%$ for $B i<0.1,4 \%$ at $B i=1.0$, and $6 \%$ at $B i=$ 100. A similar feature can be seen for the thermometers (IX) and (V). Compared with the thermometer (I), the former has double thickness in the filling material(silicon grease), and the latter is half in value in $K_{2}$. The difference is $3.6 \%$ at $B i=0.1,12 \%$ at $B i$ $=1.0$, and $14 \%$ at $B i=100$. These features indicate that the increase in thickness of the second cylinder and the decrease in thermal conductivity of the second cylinder exert an 
approximately equivalent effect on $\lambda_{1}$.

When air is used as the filling material instead of silicon grease, the response characteristics change greatly as show in Fig. 2 . So they do, even when the thickness becomes $1 / 5$ of that of the thermometer (I) as in the thermometer $(\mathrm{X})$. This is because air insulates much more than silicon grease for heat sink. The dependence of $\lambda_{1}$ on the thickness of air is similar to the case of silicon grease.

\section{Comparison between the model and the experiment}

It needs $B i$ or the mean thermal emissivity $h$ in the experiment to compare the model with the experiment. There are, however, two difficulties in evaluating $h$ : one is the effect of turbulence, which can raise $h$ as much as about 50\% (McAdams, 1954), and the other is that of the film temperature. Since $h$ depends upon fluid viscosity, which changes with time in the experiment of indicial response, $h$ can not be maintained constant. (The changes of $h$ in air and water inverse to each other because viscosity increases with temperature in air but decreases in water.) For these reasons, we roughly estimate $h$ and adopt the value at $20^{\circ} \mathrm{C}$ with no turbulence.

When a wind of $6 \mathrm{~m} / \mathrm{s}$ blows perpendicularly to the cylinder of $1 \mathrm{~cm}$ diameter, the Reynolds number becomes 3800 and the corresponding value of $h$ is $66 \mathrm{kcal} / \mathrm{m}^{2} \mathrm{hrK}$ (Eckert and Drake, 1951) or $75 \mathrm{kcal} / \mathrm{m}^{2} \mathrm{hrK}$ (McAdams, 1954). We adopt these values for the reasons mentioned above, though a wind of $6 \mathrm{~m} / \mathrm{s}$ blow parallel to the thermometer in the experiment by Tokuue et al.(1984). Then $B i$ takes the value of $0.024-0.027$ for the prototype thermometer(I). On the other hand, in the experiment in water the relative of water was referred to. So, we guess it about $10-30 \mathrm{~cm} / \mathrm{s}$. Then the Reynolds number becomes $1000-3000$ and $h=160-280 \mathrm{kcal} /$ $m^{2} h r K$ (McAdams, 1954), which makes $B i$ 0.057-0.10 for the prototype thermometer(I).

Since the time of one-term approximation is shorter than the e-folding time, $t_{1}<\tau$, in the experiment as seen in Fig. $1, \lambda_{1}$ can be calculated from Eq.(21) as $30 \mathrm{sec}$ at $\mathrm{Bi}$ 0.024-0.027 (in water), and $126 \mathrm{sec}$ at $\mathrm{Bi} \sim$ 0.057-0.10 (in air), respectively. The thermometers whose second cylinder consists of air cannot have less $\lambda_{1}$ that $31 \mathrm{sec}$ even if the thickness is as thin as $0.1 \mathrm{~mm}$. Hence we here do not treat these ones.

On the other hand, the thermometers, (I)-(IX), the filling materials of which are silicon grease, satisfy both relations between $B i$ and $\lambda$ as seen in Fig. 2, the differences of $\lambda_{1}$ among them being very small in the region of $B i<0.1$, for instance less than $1 \%$ at $B i=0.05$. In addition, their range of $\lambda$ falls within the range caused by the estimation error of $B i$, and hence we cannot identify the type of thermometer most closly similar to that used in the experiment from a comparison of $\lambda_{1}$.

Nevertheless, these facts reveal to us important characteristics, that is, response speed of the model electric resistance thermometer cannot be improved much even if the parameters are doubled or halved, so long as $B i<0.1$. For example, if another kind of stainless steel such as AISI 420 or 430 is used instead of the $18 \cdot 8$ sainless steel(AISI $304)$, the thermal conductivity increases by about $50 \%$, and the density and specific heat scarcely change (Lewis, 1977). The improvement of the e-folding time; however, exhibits no more than $1 \%$ at $B i=0.02$.

In comparing the model and the experiment in regard to $C_{1}$ we use the center value $C_{1}(0)$ instead of the areally averaged value, because there is virtually no difference between the two values in the small $B i$ region as has been stated. For simplicity we will write $C_{1}(0)$ as $C_{1}$. If the standard errors in Characteristic- 2 are brought about by the dispersion of $C_{1}$, then the values of $C_{1}$ can be estimated as $1.12 \pm 0.04$ in water and 1.11 \pm 0.05 in air. Of all the models there is no thermometer with $C_{1}$ agreeing with the experimental value in air, while there are some with $C_{1}$ 's agreeing with the experimental value in water. Even if $B i$ in air is doubled, the increase of $C_{1}$ is too little. This disagreement may be ascribed to the fact that the 
model thermometers may be too simplified, compared with the actual thermometer, though there is good agreement for $\lambda_{1}$. We assume, however, that there are some models whick intrinsically represent the actual thermometer.

The e-folding time in air is greater by 5 times than that in water, so that the values of $C_{1}$ cannot similar to each other, for, in the range of $B i<0.1, C_{1}$ is an increasing function of $B i$ for the silicon grease thermometers as seen in Fig. 4, and the difference in $B i$ by 3 times is expected to make the difference in $C_{1}$ about several percent. If we estimate $C_{1}$ in air by a slightly less value as $1.05,94 \%$ of $C_{1}$ in water, then three thermometers (V), (VII), and (IX) agree with the experiments both in air and in water.

In the thermometers (V) and (VII) the thermal conductivities of the filling material are half as great as that of the silicon grease used in the model (I). Since the thermal conductivities of the silicon grease for heat sink are not so wide range, neither of the thermom eters are good as the model of the electric resistance thermometer, the filling material of which is made of silicon grease. On the other hand, the thermometer (IX) has the same thermal conductivity in the filling material as the silicon grease for heat sink, so that the thermometer (IX) is the most closly similar model.

\section{Discussion}

As shown above, an insulating second cylinder makes $C_{1}$ a decreaing function of $B i$ over $B i c r$, which penomenon is an intrinsic feature of a composite cylinder, because this is not seen in a solid cylinder. In every model thermometer $\lambda_{1}$ is nearly half that of a solid cylinder at Bicr (see Figs. 2 and 3). The temperature profile in the second cylinder is expected to drastically change when $B i$ exceeds Bicr. Consider the thermometer (I), whose Bicr is about 0.7. The temperature difference between the inner and outer surfaces of the second cylinder, i.e., $r=a$ and $r=b$, is very small at $B i=0.01$, and still smaller at $B i=0.1$, but it shows a very large value at $B i=1.0$ as shown in Fig. 5. At $B i=1.0$ the temperature change at the outer surface $(r=$ $b$ ) already decreases below $37 \%$ when the temperature change at the inner surface $(r=$ a) reaches $90 \%$. In a solid cylinder such a large temperature difference does not occur even if $B i$ is very large (see the example at $B i=10$ in Fig. 5). The large temperature difference in the second cylinder implies that the insulating material truly works as an insulator for $B i>B i c r$. Thus Bicr can be used as an index to the insulating efficiency, since smaller Bicr means better insulation.

We can induce the criterion of $B i$ for the approximation to a first order system by using the theoretical results obtained above. When $B i \rightarrow 0$, then $C_{1} \rightarrow 1, t_{1} \rightarrow 0$, and $\Theta$ can represented by the first term of Eq.(20). Expanding the eigenvalue equation (9), we obtain the first eigenvalue $\alpha$ and the $\mathrm{e}$ folding time $\tau$ :

$$
\begin{aligned}
& \alpha=\sqrt{2 B i\left(\frac{\omega}{\mu}\right)\left\{\sigma \nu a^{2}+\sigma \omega\left(b^{2}-a^{2}\right)+\mu \omega\left(c^{2}-b^{2}\right)\right\}}, \\
& \tau=\frac{\rho_{1} S_{1} a^{2}+\rho_{2} S_{2}\left(b^{2}-a^{2}\right)+\rho_{3} S_{3}\left(c^{2}-b^{2}\right)}{2 h c}
\end{aligned}
$$

where $\rho_{j}$ and $S_{j}$ represent the density and the specific heat of $j$-th cylinder, respectively: Eq.(23) is identical with the e-folding time derived from the first order system, in which the governing equation is represented by

$$
D\left(\frac{d \Theta}{d t}\right)+2 \pi h c \Theta=0,
$$

Where $D$ is the total heat capacity of the thermometer. Thus the approximation to the 


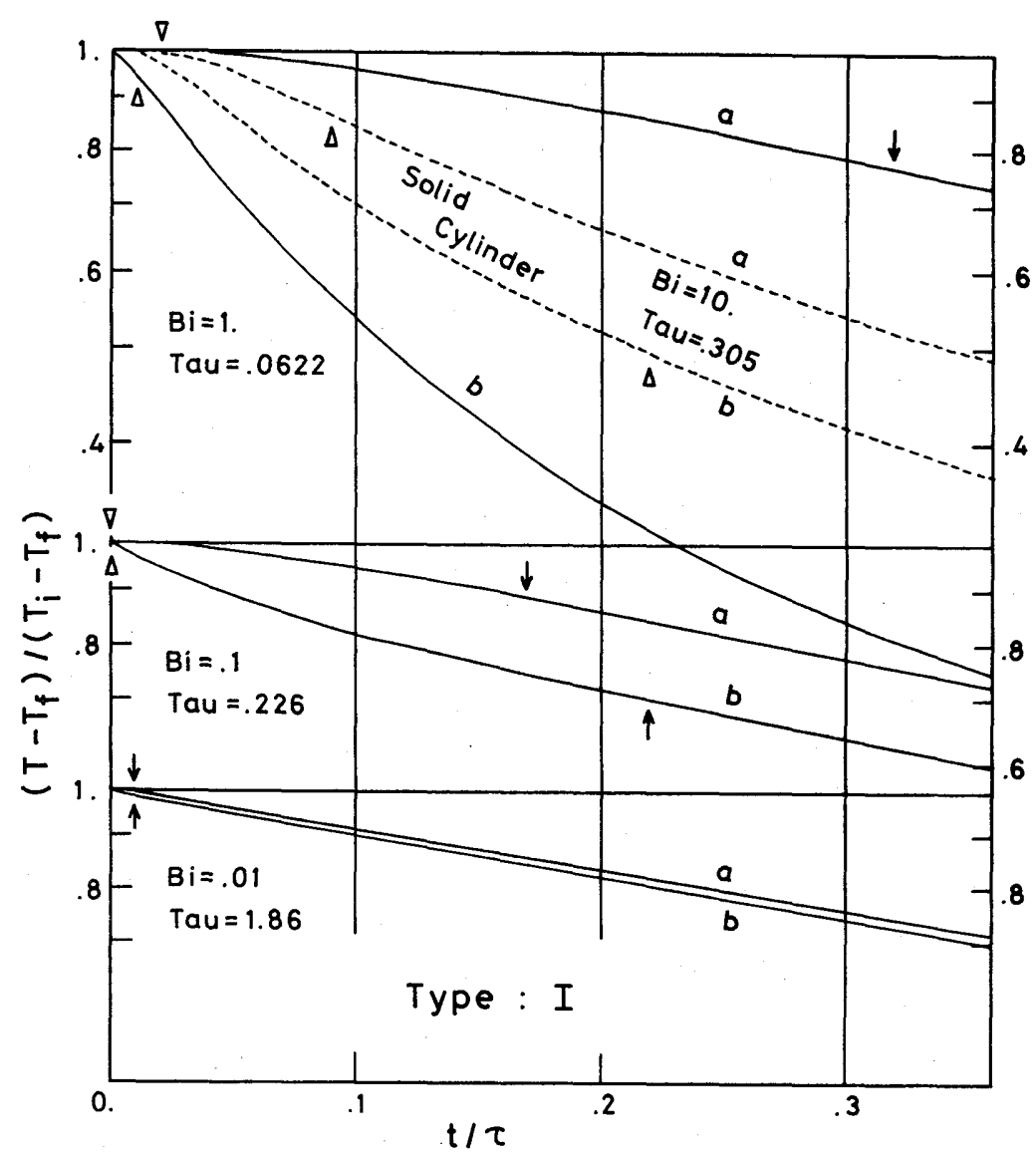

Fig. 5 Dimensionless temperature ratios at the inner(a) and outer(b) surfaces of the second cylinder for the thermometer (I) (solid curves) and a solid cylinder(dotted curves). Numbers near the curves represent $B i$. Symbols and absicca are the same as in Fig. 4 . Tau $(=\tau)$ is the value at $r=0$ and represented by the Fourie number.

first order system can be applied only to the cases where the cooling (or heating) speed (i.e., $B i)$ is so small that the eigenvalue $\alpha$ approximately satisfies Eq.(22). We do not quantitatively derive the criterion of $B i$ for the first order system, because the criterion depends upon the degree of accuracy in the approximation.

The insulating material of the second cylinder does not always works as an insulator, but shows conducting properties for small Bi. Generally speaking, non-metallic materials have smaller heat capacities than metals. Thus, when $B i$ is small enough and Eq.(23) is valid, the e-folding time of the composite cylinder which includes nonmetallic materials can be smaller than that of a metal composite cylinder. In fact, the thermometers (X)-(XIII), in which the second cylinder are filled with air, have smaller efolding times than that of solid cylinder, and the ratios of their e-folding times to that of a solid cylinder at $B i=0.001$ are $0.98,0.94$, 0.88 , and 0.76 , respectively. On the contrary, when the second cylinder is filled with silicon grease as in the thermometers (I)-(IX), $\lambda_{1}$ does not cross that of a solid cylinder as $B i \rightarrow 0$, but asymptotically approaches it. 


\section{Summary}

To account for the experimental characteristic of the Platinum electric resistance thermometer that it does not follow a first order system in usual meteorological conditions, we used a simplified model thermometer and obtained its indicial response by analytically solving its thermal diffusion equation. The model of the electric resistance thermometer is composed of three concentric cylinders of infinite length, i.e., protector sheath, filling materials, and electric resistance element. The first and third cylinders are made of conducting materials, while the second one is made of insulating material. Various thermometers, which differ in the physical properties of the filling material, are used in order to investigate the effects of the protector sheath, filling material and resistance element on the response speed of the thermometer.

The major results are as follows. (1) After a certain time (over a certain Fourier number) the solution can be approximated with one term of the exponential function, $C \exp (-t / \lambda)$, which needs two parameters, coefficient $C(>1)$ and exponent $\lambda$. (2) When $B i$ is small, the response speed hardly depends upon the inner structure. It becomes, however, sensitive to the thickness and thermal properties of the filling material as $B i$ increases. (3) The thermal properties of the protector sheath and electric resistance element have little effect on the response speed, so long as the filling material is thermally in sulating.

\section{Appendix I}

We will solve the diffusion equation of three composite cylinders by the same method as used in the case of two composite cylinders (Kataoka, 1933). The definitions of the terms and functions are the same as those in the text. Let $\Phi_{j}=T_{j}-T_{i(j=1,2,3)}$ and $\Phi_{f}=T_{f}-T_{i}$, then Eqs.(1)-(5) become

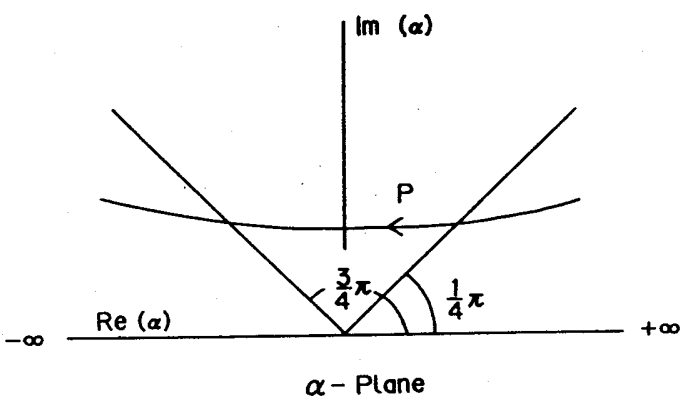

Fig. A-1 Integral path $p$ in $\alpha$-plane.

$\frac{\partial \Phi_{j}}{\partial t}=\kappa_{j}\left(\frac{\partial^{2}}{\partial r^{2}}+\frac{1}{r} \frac{\partial}{\partial r}\right) \Phi_{j}, \quad j=1,2,3$,

$(\mathrm{A}-1 \mathrm{a}, \mathrm{b}, \mathrm{c})$

$\Phi_{j}=0, \quad$ when $t=0, \quad j=1,2,3, \quad(\mathrm{~A}-2 \mathrm{a}, \mathrm{b}, \mathrm{c})$

$\Phi_{j}=\Phi_{j+1}, \quad$ at $r=r_{j}, \quad j=1,2, \quad(\mathrm{~A}-3 \mathrm{a}, \mathrm{b})$

$K_{j} \frac{\partial \Phi_{j}}{\partial r}=K_{j+1} \frac{\partial \Phi_{j+1}}{\partial r}, \quad$ at $r=r_{j}, \quad j=1,2$,

$(A-4 a, b)$

$K_{3} \frac{\partial \Phi_{3}}{\partial r}=h\left(\Phi_{f}-\Phi_{3}\right), \quad$ at $r=r_{3}$.

First, we consider the system in which the boundary condition at $r=r_{3}$ is not a radiative type as Eq.(A-5) but a fixed type as

$$
\Phi_{3}=\Phi_{f} .
$$

Then, the solutions are

$$
\begin{array}{r}
\Phi_{1}=A_{1} J_{0}(\nu \alpha r) \exp \left(-\kappa_{2} \alpha^{2} t\right), \quad(\mathrm{A}-7 \mathrm{a}) \\
\Phi_{2}=\left\{A_{2} J_{0}(\alpha r)+B_{2} Y_{0}(\alpha r)\right\} \exp \left(-\kappa_{2} \alpha^{2} t\right), \\
(\mathrm{A}-7 \mathrm{~b}) \\
\Phi_{3}=\left\{A_{3} J_{0}(\mu \alpha r)+B_{3} Y_{0}(\mu \alpha r)\right\} \exp \left(-\kappa_{2} \alpha^{2} t\right), \\
(\mathrm{A}-7 \mathrm{c})
\end{array}
$$

The boundary conditions of Eqs.(A-3a, b) and $(\mathrm{A}-4 \mathrm{a}, \mathrm{b})$ give a relation among the coefficients $A_{j}$ and $B_{j}$,

$$
\begin{aligned}
& D^{t}\left(A_{2}, B_{2}, A_{3}, B_{3}\right) \\
& \quad=A_{1}{ }^{t}\left(J_{0}(\nu \alpha a), J_{1}(\nu \alpha a), 0,0\right),
\end{aligned}
$$

where superscript $t$ denotes a transpose operator and $D$ is a $4 \times 4$ matrix, 


$$
D=\left(\begin{array}{cccc}
J_{0}(\alpha a) & Y_{0}(\alpha a) & 0 & 0 \\
\omega J_{1}(\alpha a) & \omega Y_{1}(\alpha a) & 0 & 0 \\
J_{0}(\alpha b) & Y_{0}(\alpha b) & -J_{0}(\mu \alpha b) & -Y_{0}(\mu \alpha b) \\
\sigma J_{1}(\alpha b) & \sigma Y_{1}(\alpha b) & -J_{1}(\mu \alpha b) & -Y_{1}(\mu \alpha b)
\end{array}\right) .
$$

If we introduce $A_{1}$ as

$$
A_{1}=\frac{\operatorname{Det}(D)}{U(r, \alpha)}
$$

where

$$
U(r, \alpha)=V(\alpha) P(r, b, \mu \alpha)-\sigma W(\alpha) O(r, b, \mu \alpha),
$$

then we can obtain $A_{2}, B_{2}, A_{3}$ and $B_{3}$ from Eq.(A-9). Inserting them into Eq.(A-7) and reforming $\Theta_{1}, \Theta_{2}$ and $\Theta_{3}$ so as to satisfy the initial and boundary conditions, i.e., Eqs. $(\mathrm{A}-2 \mathrm{a}, \mathrm{b}, \mathrm{c})$ and Eq. $(\mathrm{A}-6)$, we have the solutions

$$
\begin{aligned}
& \Phi_{1}=\frac{\Phi_{f}}{i \pi} \int_{p} \frac{\omega P(a, a, \alpha) P(b, b, \mu \alpha)}{\alpha U(c, \alpha)} J_{0}(\nu \alpha r) \exp \left(-\kappa_{2} \alpha^{2} t\right) d \alpha \\
& \Phi_{2}=\frac{\Phi_{f}}{i \pi} \int_{p} \frac{P(b, b, \mu \alpha)}{\alpha U(c, \alpha)} V(r, a, \alpha) \exp \left(-\kappa_{2} \alpha^{2} t\right) d \alpha, \\
& \Phi_{3}=\frac{\Phi_{f}}{i \pi} \int_{p} \frac{U(r, \alpha)}{\alpha U(c, \alpha)} \exp \left(-\kappa_{2} \alpha^{2} t\right) d \alpha,
\end{aligned}
$$

where the integral path $p$ is shown in Fig. A-1. These solutions can be easily proved to satisfy the initial conditions of Eqs. $(A-2 a, b, c)$ and the boundary conditions of Eqs. $(A-3 a, b),(A-4 a, b)$ and $(A-6)$.
Next we return to the case whose boundary condition at $r=r_{3}$ is the radiative type given by Eq.(A-2). It follows from Eqs. $(A-12 a, b, c)$ that the solutions are

$$
\begin{aligned}
& \Phi_{1}=\frac{\Phi_{f}}{i \pi} \int_{p}^{\alpha\left[\frac{K_{3}}{h} \frac{\partial U(r, \alpha)}{\partial r}+U(r, \alpha)\right]_{r=c}} J_{0}(\nu \alpha r) \exp \left(-\kappa_{2} \alpha^{2} t\right) d \alpha, \\
& \Phi_{2}=\frac{\Phi_{f}}{i \pi} \int_{p}^{\alpha\left[\frac{K_{3}}{h} \frac{\partial U(r, \alpha)}{\partial r}+U(r, \alpha)\right]_{r=c}} V(r, a, \alpha) \exp \left(-\kappa_{2} \alpha^{2} t\right) d \alpha, \\
& \Phi_{3}=\frac{\Phi_{f}}{i \pi} \int_{p}^{\alpha\left[\frac{K_{3}}{h} \frac{\partial U(r, \alpha)}{\partial r}+U(r, \alpha)\right]_{r=c}} \exp \left(-\kappa_{2} \alpha^{2} t\right) d \alpha,
\end{aligned}
$$

where the integral path $p$ is the same as that in Eqs. $(\mathrm{A}-11 \mathrm{a}, \mathrm{b}, \mathrm{c})$. These solutions can also be proved to meet the initial conditions of Eqs. $(A-2 a, b, c)$ and the boundary conditions of Eqs. $(\mathrm{A}-3 \mathrm{a}, \mathrm{b}),(\mathrm{A}-4 \mathrm{a}, \mathrm{b})$. Since at $r=c$ the integral path $p$ may be replaced by the one around the origin, $q$, it follows that 


$$
K_{3} \frac{\partial \Phi_{3}}{\partial r}+h \Phi_{3}=\frac{h \Phi_{f}}{i 2 \pi} \oint_{q} \frac{\exp \left(-\kappa_{2} \alpha^{2} t\right)}{\alpha} d \alpha=h \Phi_{f}
$$

Thus the boundary condition of Eq. $(A-5)$ is also satisfied.

The function in Eqs.(A-13) are even functions of $\alpha$ and have poles only on the real axis (see appendix II). Hence, evaluating the integral by residues and rewriting the solutions from $\Phi$ to $\Theta$, we have

$$
\begin{aligned}
& \Theta_{1}=-2 \sum_{n} \frac{\omega P(a, a, \alpha) P\left(b, b, \mu \alpha_{n}\right)}{\alpha_{n} D N\left(\alpha_{n}\right)} J_{0}\left(\nu \alpha_{n} r\right) \exp \left(-\kappa_{2} \alpha_{n}{ }^{2} \mathrm{t}\right), \\
& \Theta_{2}=-2 \sum_{n} \frac{P\left(b, b, \mu \alpha_{n}\right) V\left(r, a, \alpha_{n}\right)}{\alpha_{n} D N\left(\alpha_{n}\right)} \exp \left(-\kappa_{2} \alpha_{n}{ }^{2} \mathrm{t}\right), \\
& \Theta_{3}=-2 \sum_{n} \frac{U\left(r, \alpha_{n}\right)}{\alpha_{n} D N\left(\alpha_{n}\right)} \exp \left(-\kappa_{2} \alpha_{n}{ }^{2} \mathrm{t}\right),
\end{aligned}
$$

with

$$
D N(\alpha)=\left(\frac{\partial}{\partial \alpha}\right)\left[\frac{K_{3}}{h} \frac{\partial U(r, \alpha)}{\partial r}+U(r, \alpha)\right]_{r=c}
$$

where $\alpha_{n}$ is the $n$-th root of the eignevalue equation

$$
\left[\frac{K_{3}}{h} \frac{\partial U(r, \alpha)}{\partial r}+U(r, \alpha)\right]_{r=c}=0 .
$$

\section{Appendix II}

\section{II-A The eigenvalue equation (A-16) has} no complex root $\xi+i \eta(\xi \eta \neq 0)$.

If we define $U_{1}, U_{2}$ and $U_{3}$ in the ranges $[0, a], \quad[a, b]$ and $[b, c]$, respectively, as

$$
\begin{aligned}
U_{1}(r, \alpha)= & \omega P(b, b, \mu a) P(a, b, \alpha) J_{0}(\nu \alpha r), \\
U_{2}(r, \alpha)= & P(b, b, \mu a) V(r, a, \alpha), \quad(\mathrm{A}-17 \mathrm{a}) \\
U_{3}(r, \alpha)= & V(b, a, \alpha) P(r, b, \mu \alpha) \\
& -\sigma W(b, a, \alpha) O(r, b, \mu \alpha),
\end{aligned}
$$

real axis (see appendix II). Hence, evaluating the integral by residues and rewriting the solutions from $\Phi$ to $\Theta$, we have

$$
\frac{\partial}{r \partial r}\left(r \frac{\partial U_{j}}{\partial r}\right)+\varepsilon_{j}{ }^{2} \alpha^{2} U_{j}=0, \quad j=1,2,3,
$$

(A-18a,b,c) where $\left(\epsilon_{1}, \epsilon_{2}, \epsilon_{3}\right)=(\nu, 1, \mu)$. Let $V_{j}=$ $U_{j}(r, \beta),{ }_{j=1,2,3}$, then Eqs.(A-18a, b, c) and the corresponding equations for $V_{j}$ yield

$$
\begin{aligned}
& \left(\frac{\nu}{\omega}\right)\left(\beta^{2}-\alpha^{2}\right) \int_{0}^{a} r U_{1} V_{1} d r=\left(\frac{1}{\nu \omega}\right)\left[r V_{1} \frac{\partial U_{1}}{\partial r}-r U_{1} \frac{\partial V_{1}}{\partial r}\right]_{0}^{a}, \\
& \left(\beta^{2}-\alpha^{2}\right) \int_{a}^{b} r U_{2} V_{2} d r=\left[r V_{2} \frac{\partial U_{2}}{\partial r}-r U_{2} \frac{\partial V_{2}}{\partial r}\right]_{a}^{b},
\end{aligned}
$$




$$
\left(\frac{\mu}{\sigma}\right)\left(\beta^{2}-\alpha^{2}\right) \int_{b}^{c} r U_{3} V_{3} d r=\left(\frac{1}{\mu \sigma}\right)\left[r V_{3} \frac{\partial U_{3}}{\partial r}-r U_{3} \frac{\partial V_{3}}{\partial r}\right]_{b}^{c} .
$$

Since Eqs (A-17a, b, c) lead to the following relations:

$$
\begin{array}{ll}
U_{1}=U_{2}, & \left(\frac{1}{\nu \omega}\right) \frac{\partial U_{1}}{\partial r}=\frac{\partial U_{2}}{\partial r}, \quad \text { at } r=a, \\
U_{2}=U_{3}, & \left(\frac{1}{\mu \sigma}\right) \frac{\partial U_{2}}{\partial r}=\frac{\partial U_{3}}{\partial r}, \quad \text { at } r=b,
\end{array}
$$

and similar equations hold for $V_{j}$, the summation of Eqs.(A-19a, b, c) yields

$$
\begin{aligned}
& \left(\beta^{2}-\alpha^{2}\right)\left\{\left(\frac{\nu}{\omega}\right) \int_{0}^{a} r U_{1} V_{1} d r+\int_{a}^{b} r U_{2} V_{2} d r+\left(\frac{\mu}{\sigma}\right) \int_{b}^{c} r U_{3} V_{3} d r\right\} \\
= & \left(\frac{c}{\mu \sigma}\right)\left[r V_{3} \frac{\partial U_{3}}{\partial r}-r U_{3} \frac{\partial V_{3}}{\partial r}\right]_{r=c} .
\end{aligned}
$$

Let $\alpha$ and $\beta$ be roots of Eq.(A-16), than

$$
\frac{\partial U_{3}}{\partial r}=-\left(\frac{h}{K_{3}}\right) U_{3}, \quad \text { at } r=c,
$$

and the same equation holds for $V_{3}$. Substi-

$V_{3}$ into Eq.(A-21) we have. tuting Eqs.(A-22) and the same equation for

$$
\left(\beta^{2}-\alpha^{2}\right)\left\{\left(\frac{\nu}{\omega}\right) \int_{0}^{a} r U_{1} V_{1} d r+\int_{a}^{b} r U_{2} V_{2} d r+\left(\frac{\mu}{\sigma}\right) \int_{b}^{c} r U_{3} V_{3} d r\right\}=0 .
$$

Eq.(A-23) implies that $\alpha$ and $\beta$ cannot be complex conjugate to each other. The reason for this is as follows. If $\alpha$ and $\beta$ are complex conjugate to each other, so are $U_{i}$ and $V_{i}$. Concequently, all the integrals in Eq. (A-23) become positive, and the lefthand side of Eq.(A-23) also becomes non-zero, which rusult contradicts with Eq.(A-23).

\section{II-B The eigenvalue equation (A-16) has no pure imaginary root.}

(i) Let define a function

$$
y_{n}(x)=\frac{Y_{n}(i x)}{J_{n}(i x)}-i, \quad n=0,1,2,3 \cdots
$$

then $Y_{n}$ is a real function when $x$ is real (see Carslaw (1921) in the case of $n=0$ ). Its derivative is given by

$$
\left(\frac{d}{d x}\right) y_{n}=i\left[J_{n}(t) \frac{d Y_{n}(t)}{d t}-Y_{n}(t) \frac{d J_{n}(t)}{d t}\right]_{t=i x} / J_{n}{ }^{2}(i x)=\frac{2}{\pi x J_{n}{ }^{2}(i x)} .
$$

We consider hereafter that $x$ is positive real. Then we get from Eq.(A-25) $y_{n}(x)= \begin{cases}\text { monotonically increasing, } & n: \text { even, } \\ \text { monotonically decreasing, } & n: \text { odd, }\end{cases}$ (A-26) because $J_{n}^{2}(i x)>0(<0)$ for even (odd) $n$. Since from Eq.(A-24) $Y_{n} \rightarrow 0$ as $x \rightarrow \infty$, Eq.(A-26) yields

$y_{n}(x)=\left\{\begin{array}{cc}<0, & n: \text { even } \\ >0, & n: \text { odd } .\end{array}\right.$ 
(ii) Let $a, b(>a), z, \xi$ be positive reals, and a then positive definite function be noted by $P D$,

$$
\begin{aligned}
& O(b, a, i z)=J_{0}(i b z) J_{0}(i a z)\left\{y_{0}(a z)-y_{0}(b z)\right\}=-P D, \\
& P(b, a, i z)=J_{0}(i b z) J_{1}(i a z)\left\{y_{1}(a z)-y_{0}(b z)\right\}=-P D,
\end{aligned}
$$

because $J_{0}(i \xi)=P D, J_{1}(i \xi)=i P D, y_{0}(a z)<y_{0}(b z)$

$$
V(i z)=i P D
$$

from Eq.(A-26), $y_{1}(\xi)>0$ and $y_{0}(\xi)<0$ from Eq.(A-27). Similarly, we can prove

$$
W(i z)=-P D .
$$

$$
\begin{aligned}
& \begin{array}{l}
P(a, b, i z)=i P D, \\
\begin{array}{c}
(\mathrm{A}-30) \\
(\mathrm{A}-31)
\end{array} \\
\left(\frac{K_{3}}{h}\right)\left[\frac{\partial U(r, i \alpha)}{\partial r}+U(r, i \alpha)\right]_{r=c} \\
\text { from Eqs. }(\mathrm{A}-28)-(\mathrm{A}-33) \text { that }
\end{array} \\
& =-\left(\frac{K_{3}}{h}\right) i \mu \alpha\{V(i \alpha) Q(c, b, i \mu \alpha)+\sigma W(i \alpha) P(b, c, i \mu \alpha)\} \\
& \quad+V(i \alpha) P(c, b, i \mu \alpha)-\sigma W(i \alpha) O(c, b, i \mu \alpha)
\end{aligned}
$$

(iii) Let $\alpha$ be positive real, then it follows

Eq.(A-34) means that the eigenvalue equation (A-16) has no positive pure imaginary root. As a result, the eigenvalue equation (A-16) has no negative pure imaginary root either, because Eq. $(\mathrm{A}-16)$ is a polynomial function of real coefficients.

\section{References}

Carslaw, H. S., 1921: Mathematical theory of the conduction of heat in solids. 2nd ed., Macmillan and Co. Limited, London, $268 \mathrm{pp}$.

Carslaw, H. S. and Jaeger, J. C., 1958: Conduction of heat in solids. 2nd ed., Clarendon Press, Oxford, $510 \mathrm{pp}$.

Eckert, E. R. G. and R. M. Drake, 1951: Heat and mass transfer. 2nd ed., McGraw-Hill, New York, $530 \mathrm{pp}$.

Meteorological Office, 1956: Handbook of meteorological instruments. Part 1. M.O. 577, Her
Majesty's Stationery Office, London, 458 pp. Heisler, M. P., 1947: Temperature chart for induction and constant-heating. Trans. ASME, 69, 227-236.

Lewis, J. R., 1977: Physical properties of stainless steels. Handbook of stainless steels edited by D. Peckner and I. M. Bernstein, McGraw-Hill, New York.

Kataoka, H., 1933: Conduction of heat in a cylinder composed of two different materials. Journal of Japan Society of Mechanical Engineers, 36, 31-35. (in Japanese with English abstract)

Mcadams, W. H., 1954: Heat transmission. 3rd ed., McGraw-Hill, New York, 383 pp.

Middleton, W. E. K. and A. F. Spilhaus, 1953: Meteorological Instruments. 3rd edition, University of Toronto Press, Tronto, $286 \mathrm{pp}$.

Tokuue, H., I. Okuzumi and A. Yamazaki, 1984: On the time constant of the electric resistance thermometer. Technical reports of instrument, 5906, JMA.(in Japanese) 


\section{3 層の円筒から成る抵抗温度計の過渡応答}

\section{柴田清孝}

白金抵抗温度計は一階の微分方程式の系で記述されないという実験結果を説明するため抵抗温度計のモデ ルをつくり，その熱伝導方程式を解析的に解いて過渡応答を求めた。モデルは保護管・充填物質・抵抗線層 の 3 層から成る無限長円筒と単純化されている。この結果を使って充填物質の物理特性の異なる種々のモデ ルについて過渡応答を評価した。主な結果は次の通りである。（1）過渡応答はあるフーリ工数以降で $C \exp$ $(-t / \lambda)$ と指数関数の単項式で近似できるが, 係数 $C(>1)$ と指数 $\lambda$ の 2 つパラメータが必要である。 （2）応答速度はビオ一数の小さいうちは内部構造の影響をほとんど受けないが, ビオー数が大きくなると 充填物質の厚さ，熱的性質に敏感になってくる。（3）充填物質が熱絶縁的である限り，保護管および抵抗 線層の熱的性質は応答速度にほとんど影響を与えない。 